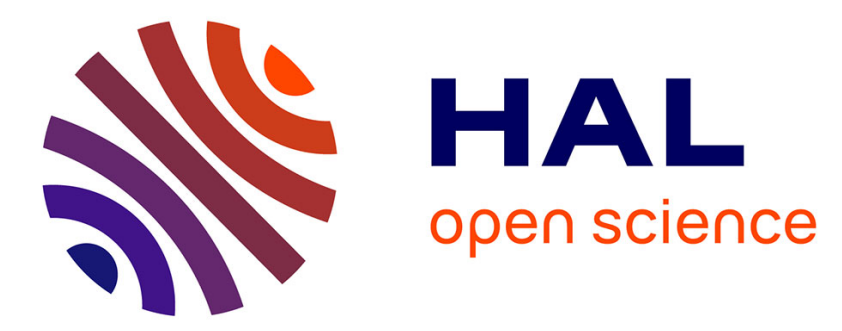

\title{
Beam-particle approach to model cracking and energy dissipation in concrete: Identification strategy and validation
}

\author{
M. Vassaux, C. Oliver-Leblond, B. Richard, F. Ragueneau
}

\section{To cite this version:}

M. Vassaux, C. Oliver-Leblond, B. Richard, F. Ragueneau. Beam-particle approach to model cracking and energy dissipation in concrete: Identification strategy and validation. Cement and Concrete Composites, 2016, 70, pp.1-14. 10.1016/j.cemconcomp.2016.03.011 . hal-01297333

\section{HAL Id: hal-01297333 \\ https://hal.sorbonne-universite.fr/hal-01297333}

Submitted on 4 Apr 2016

HAL is a multi-disciplinary open access archive for the deposit and dissemination of scientific research documents, whether they are published or not. The documents may come from teaching and research institutions in France or abroad, or from public or private research centers.
L'archive ouverte pluridisciplinaire HAL, est destinée au dépôt et à la diffusion de documents scientifiques de niveau recherche, publiés ou non, émanant des établissements d'enseignement et de recherche français ou étrangers, des laboratoires publics ou privés. 


\title{
Beam-Particle approach to model cracking and energy dissipation in concrete: Identification strategy and Validation
}

\author{
M. Vassaux ${ }^{\mathrm{a}}$, C. Oliver-Leblond $d^{\mathrm{a}, *}$, B. Richard ${ }^{\mathrm{b}}$, F. Ragueneau ${ }^{\mathrm{a}}$ \\ ${ }^{a}$ LMT-Cachan (ENS Cachan/CNRS/UPMC/PRES UniverSud Paris) \\ 61, avenue du Président Wilson, 94230 Cachan, France \\ ${ }^{b} C E A, D E N, D A N S, D M 2 S$, SEMT, Laboratoire d'Études de Mécanique Sismique \\ F-91191 Gif-sur-Yvette, France
}

\begin{abstract}
This paper focuses on the application of a beam-particle model to study the failure of concrete under complex loading. The formulation of the model is based both on lattice models and discrete elements models in order to capture cohesion, failure and frictional contact of the crack surfaces. To correctly describe the elastic phase, the peak load and the post-peak phase, the failure criteria is discussed and heterogeneities are introduced. The calibration of this model is detailed and illustrated. Finally, several test cases are analysed in order to validate the model.
\end{abstract}

Keywords: Beam-particle model; Cracking; Energy Dissipation; Identification

\section{Introduction}

In the context of sustainable development, the study of the cracking of concrete has become of primary importance. Continuum mechanics allow a global study of the rupture of such a material but cannot fully and finely describe the cracking process. Complex civil engineering structures may deal with numerous cracks prapagation under 3D state of stresses. Only macroscopic continuous models are available at the engineering scale, implying major drawback such as

\footnotetext{
* Corresponding author

Email address: oliver@lmt.ens-cachan.fr (C. Oliver-Leblond)
} 
a physical representation of the discrete feature of cracking and the difficulty of identification for complex loading history. On the contrary, Discrete Elements Models (DEM) - such as lattice models or particle models - are designed to describe discontinuous mediums. In spite of high computational costs, the necessity to enhance discrete element models accounting for complex loadings lies in the possibility to couple such approaches with continuous ones thanks to multiscales framework [1] and to use this physical type of models as virtual laboratory tests for better identification [2].

The first lattice model has been introduced by [3] to solve classical problems of elasticity. The elastic material is discretised using one-dimensional elements - springs or beams - that allow force transfers between the nodes forming the lattice. The development of computational simulation allowed its extension to the study of metals by considering a brittle behaviour for the lattice elements [4]. [5] and [6] modified the approach to apply it to the quasi-brittle failure of concrete in tensile mode. However, these models cannot describe the fracturing process in compressive mode.

Particle models have been proposed by [7] to study the behaviour of granular assemblies. For the first applications, only contact forces were needed to model the interactions between the particles. Then [8] proposed a modification to model cohesive interactions and thus materials such as concrete. Unfortunately, such modelling of the cohesion of granular materials generally lacks the simplicity of the lattice models and thus its computation time is much higher.

In this paper, the combined beam-particle model - inspired by [9] and modified by [10] and [11] - is used. The concrete is represented through polygonal particles linked together by brittle beams and exhibiting frictional contact after failure of the beams. With this model, it is possible to qualitatively reproduce the failure pattern for compressive or tensile simulations.

Unfortunately, this model raises the same problem as the lattice models as regards the energy dissipation. Indeed, it is well known that those models generally exhibit a brittle failure behaviour in tension [6]. To reproduce the quasi-brittle behaviour of the concrete in tension, material heterogeneities are 
introduced. The tendency of those models to exhibit a brittle behaviour in compression is less known but still a major issue. The formulation of the local criterion is discussed to overcome this difficulty.

The complete calibration process is then presented and validated. Finally, the beam-particle model is applied to various test cases of tensile, compressive and shear failure.

\section{Description of the model}

The main features of the beam-particle model are summarised. A more detailed description of the proposed model can be found in the literature $[10,11]$.

\subsection{Mesh generation}

The medium is described as an assembly of polygonal particles. The generation of the mesh follows the proposition of [12].

Firstly, a grid is superimposed on the specimen. The spacing of this grid corresponds to the average particle size $l_{p}$ and is set as a material parameter.

Secondly, points are randomly generated in each cell of the grid. Those points represent the centroids of the particles. The heterogeneity of the mesh is controlled by the random placement of the centroids of the particles.

Thirdly, Delaunay's triangulation of the set of points is performed. The edges of the Delaunay triangles represent the cohesive beams linking neighbouring particles.

Finally, the polygonal particles are computed from Voronoi's tessellation.

\subsection{Particle interactions}

\subsubsection{Cohesive forces}

In order to represent the cohesion of the material, the particles are joined together through Euler-Bernoulli beams linking the centroids of the particles. Those particles are rigid. Therefore, the cohesive forces must reproduce the elastic behaviour of the material.

The beam $i-j$, linking the particles $i$ and $j$, has four parameters (see on figure 1). Two of them, the length $l_{b, i j}$ and the cross section area $A_{b, i j}$, 
are prescribed by the mesh geometry and are different for each beam. The two other parameters, the Young's modulus of the beams $E$ and the inertia coefficient $\alpha=64 I_{b, i j} \pi / A_{b, i j}^{2}$ - where $I_{b, i j}$ is the moment of inertia - are supposed to be equal for all beams.

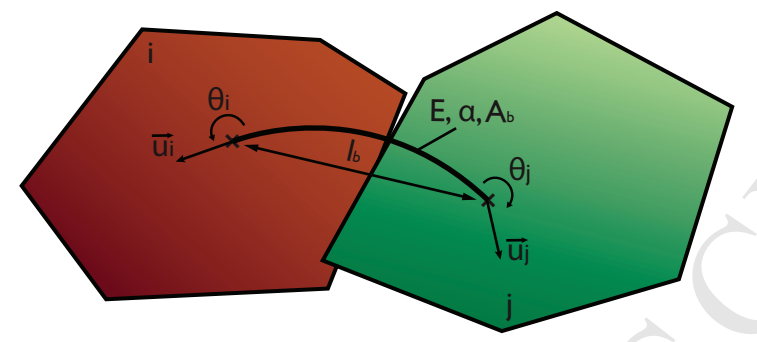

Figure 1: Two cohesively linked particles

The cohesion forces and moment between two connected particles $i$ and $j$ are expressed as:

$$
\underline{F}_{c o h, i j}=\left\{\begin{array}{l}
F_{N, i j}=\frac{E A_{b, i j}}{l_{b, i j}}\left(\underline{u}_{i}-\underline{u}_{j}\right) \cdot \underline{n}_{b, i j} \\
F_{T, i j}=\frac{12 E I_{b, i j}}{l_{b, i j}^{3}}\left(\underline{u}_{i}-\underline{u}_{j}\right) \cdot \underline{t}_{b, i j}-\frac{6 E I_{b, i j}}{l_{b, i j}^{2}}\left(\theta_{i}-\theta_{j}\right) \\
M_{Z, i j}=\frac{6 E I_{b, i j}}{l_{b, i j}^{2}}\left(\underline{u}_{j}-\underline{u}_{i}\right) \cdot \underline{t}_{b, i j}+\frac{4 E I_{b, i j}}{l_{b, i j}}\left(\theta_{i}-\frac{\theta_{j}}{2}\right)
\end{array}\right.
$$

where $\underline{u}_{i}$ and $\underline{u}_{j}$ are the displacements of the particles $i$ and $j, \theta_{i}$ and $\theta_{j}$ are the rotations of the particles $i$ and $j$ and $\underline{n}_{b, i j}$ and $\underline{t}_{b, i j}$ are the normal and tangential vectors of the cross-section of the beam $i-j$.

At this point, the discrete model is equivalent to a lattice model $[13,14]$.

\subsubsection{Frictional Contact}

The modelling of concrete failure under cyclic or multi-axial loadings requires considering frictional contact interactions between particles to capture mechanisms such as crack closure or aggregate interlocking.

Contact interactions are only introduced when two separated particles - i.e. that are not linked by a cohesive beam - overlap. The magnitude of the contact 
forces, as well as their direction and their point of application, are computed as a function of the overlapping areas because particles are perfectly rigid bodies.

Overlapping. First, it is necessary to detect the overlapping of two particles. Since particles are polygonal, the intersection cannot be analytically described — as with discs - and numerical tools are required. Overlap detection is a timeconsuming process, increasing quickly with regard to the number of particles. In order to limit its costs, intersection search is restricted to close neighbours [15].

The complete description of the polygonal intersection is computed through an algorithm developed for convex polygons [16]. Then, the following contact properties (see on figure 2) are computed:

- the intersection area $S_{r}$ which is the area of the intersection polygon.

- the contact band width $L_{c}$ which the longest segment contained in the intersection polygon.

- the contact normal vector $\overrightarrow{n_{c}}$ and tangential vector $\overrightarrow{t_{c}}$ to the longest segment in the intersection polygon.

- the application point $C$ of the contact forces which is the center of inertia of the intersection polygon.

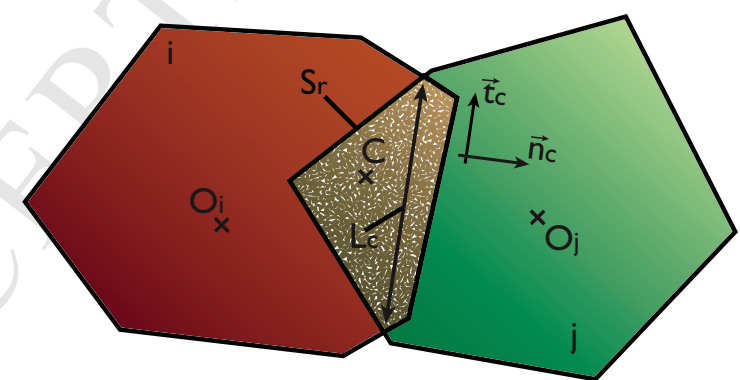

Figure 2: Two overlapping particles in contact

Constitutive contact model. The beam theory used for cohesion turns out to be very convenient for elastic contact force computation between polygonal par- 
ticles $[17,9]$. A slight modification is made in the normal force formula to introduce the overlap area $S_{r}$ :

$$
\underline{F}_{c o n t, i j}=-\frac{E S_{r, i j}}{l_{c, i j}} \underline{n}_{c, i j}
$$

where $l_{c}$ is a characteristic length supposed to be the harmonic mean of the diameter of the contacting particles:

$$
\frac{1}{l_{c}}=\frac{1}{2}\left(\frac{1}{D_{i}}+\frac{1}{D_{j}}\right)
$$

Frictional sliding. A Coulomb's type of friction is considered introducing the friction coefficient $\mu$ :

$$
\underline{F}_{f r i c, i j}=\min \left(\frac{E I_{c, i j}}{\left(l_{c, i j}\right)^{3}}\left[\left(\underline{u}_{c, i j}-\underline{u}_{c, j i}\right) \cdot \underline{t}_{c, i j}-\Delta u_{s, i j}\right], \mu|| \underline{F}_{c o n t, i j} \|\right) \underline{t}_{c, i j}
$$

where the inertia moment is $I_{c}=\frac{1 \times l_{c}^{3}}{12}$.

Before reaching perfectly plastic friction, the behaviour is elastic and computed from the tangential force formulae in beam theory. Only the fraction of the displacements accumulated since contact has begun is taken into account:

$$
\underline{u}_{c, i j}^{t+1}= \begin{cases}\underline{u}_{c, i j}^{t}+\underline{\delta u_{i}^{t}} & \text { if } i \text { and } j \text { are overlapping } \\ 0 & \text { if } i \text { and } j \text { are not overlapping }\end{cases}
$$

where $\underline{u}_{c, i j}$ is the accumulated displacement in the normal direction of the particle $i$ since the contact with particle $j$ has begun. A new internal variable $\Delta u_{s}$ measuring the sliding relative displacement between two particles is introduced and updated in order to verify Coulomb's criterion. Both internal variables $\underline{u}_{c}$ and $\Delta u_{s}$ are reset when contact is lost.

\subsection{Fracture}

A brittle behaviour is imposed to the beams in order to reproduce the quasibrittle behaviour of the material. The breaking threshold $P_{i j}$ of the beam linking particle $i$ to particle $j$ depends on the axial strain of the beam and on the rotations of the two particles: 


$$
P_{i j}\left(\frac{\epsilon_{i j}}{\epsilon_{i j}^{c r}}, \frac{\left|\theta_{j}-\theta_{i}\right|}{\theta_{i j}^{c r}}\right)>1
$$

where the breaking parameters are $\epsilon_{i j}^{c r}$, the critical strain of the beam $i-j$, and $\theta_{i j}^{c r}$, the critical rotation of the beam $i-j$.

When the breaking threshold is reached, the beam is removed and a crack appears at the common edge of the particles formerly linked by this beam. A local crack opening $e_{i j}$ can then be computed by considering the relative displacements of those particles.

\subsection{Implicit solving algorithm}

Unlike most DEM models, the solving algorithm developed for the beamparticle model is implicit [11]. In the context of latter pursued analysis, only quasi-static loadings are considered at the macroscopic scale, therefore dynamic effects are consistently neglected at the lower scale, including inertial cracking effects [18]. Neglecting dynamic effects allows us to avoid the introduction of local kinematics displacements field assumption at the RVE scale. It also allows interesting computation costs reduction, and consequently permits implicit integration. Robust integration is made possible by means of a combination of the saw-tooth algorithm, of a predictor-corrector algorithm and finally of a numerical relaxation.

The saw-tooth algorithm proposed by [19] is modified to compute the failure of the cohesive beams. If a beam exceeds its failure criterion after reaching equilibrium, the beam is removed and equilibrium is solved again until no more beams fail. This way, the stress redistribution due to the relaxation of the forces contained in the broken beam is automatically performed. If more than one beam exceeds its failure criterion, the beam to be removed is the one which exceeds its criterion most.

The iterative predictor-corrector algorithm is employed in between the sawtooth iterations to compute the equilibrium of the cohesion forces, the contact forces and the frictional forces. Unlike cohesion, the contact behaviour is strongly non-linear and dependent on the displacements and rotations of the 
particles. The non-linearities lead to robustness issues and a predictor step is mandatory to improve convergence. An efficient prediction of the equilibrium is to assume a linear dependence between the displacements and the contact forces, using an equivalent secant stiffness. As a result cohesion forces, contact forces and frictional forces can be assessed simultaneously during the predictor step.

Relaxation is done by only considering a certain amount of the predicted displacement increment, if the residue increases or decreases too slowly:

$$
\underline{u}^{k+1}=\underline{u}^{k}+h^{k+1} \delta \underline{u}^{k+1} \quad h^{k+1}= \begin{cases}h^{k} & \text { if } \delta \underline{r}^{k+1} \leq 10^{-4} \\ \frac{h^{k}}{2} & \text { if } \delta \underline{r}^{k+1}>10^{-4}\end{cases}
$$

where $\underline{\delta u^{k+1}}$ is the predicted increment, $\underline{r}^{k+1}$ is the residue increment and $h^{k+1}$ is the relaxation factor at the current iteration $k+1$.

\section{Failure behaviour and energy dissipation}

Using a lattice discrete model with a simple brittle behaviour for the beams allows a straightforward modelling of the breaking patterns. Nevertheless, those models are limited in terms of energy dissipation, as they exhibit a brittle failure behaviour of the material [9]. Moreover, the energy dissipation is dependent on the mesh size [6].

In tension, energy dissipation during the post-peak regime is explained by the occurrence of crack-bridging, due to the presence of heterogeneities. In the case of concrete, defects, pores or interfaces would be a preferable path of crack propagation which will result in a more dissipative path than in a homogeneous material. For that reason, it has been hinted that a mesoscopic description of the material - reproducing the different phases - is required to obtain a more realistic dissipation $[20,21]$. However, a mesh based on the meso-structure implies the introduction of lower-scale material parameters which would significantly complicate the model calibration. Here, we will simply introduce heterogeneities through statistical distributions of the breaking parameters as proposed by [22]. 
To correctly reproduce the tensile behaviour and ensure the mesh objectivity, several authors have proposed to include a softening behaviour into the failure model $[23,24]$. We show in this section that it is not necessary to implement such laws in order to capture the quasi-brittle behaviour of the material. Indeed, the introduction of heterogeneities of the breaking parameters seems to be sufficient. Moreover, the density of the mesh is set as a material parameter which means that mesh objectivity is beyond the scope of this model.

Post-peak behaviour in compression is not as much influenced by heterogeneities and material properties as in tension. The relative ductility of the compression behaviour is rather explained by structural effects. The different mechanisms occurring during compression tests, according to [25] or [26], are summarized on figure 3. First, cracking is diffuse and occurs only at the microscale. Then, Poisson's effect induces extensions in the perpendicular direction to the applied load and cracks propagate in the direction of this load. Columns of material are then created but there is no significant loss of stiffness. Finally, peak load is reached and the cracks start to localize. The columns previously created collapse under excessive bending, leading to the failure of the specimen. In order to account for such mechanisms, we focus our interest on the formulation of the local failure criterion.

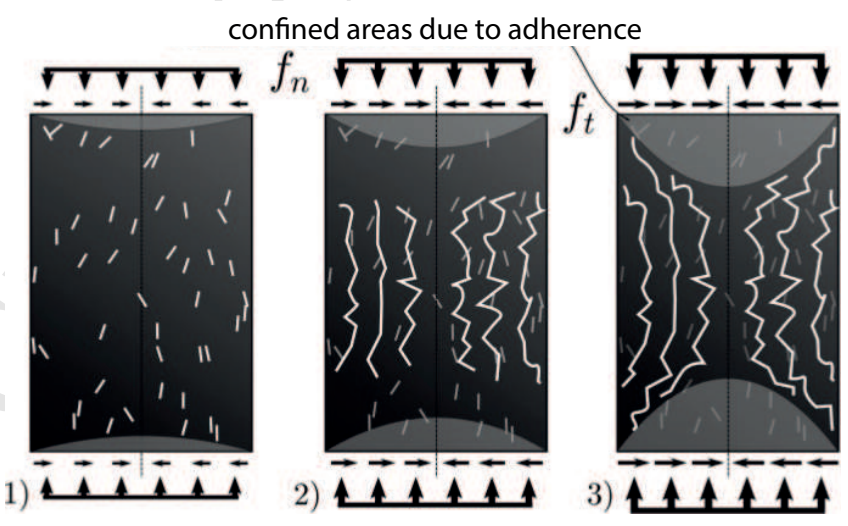

Figure 3: Three stages of failure under compression 


\subsection{Statistical aspects}

In order to reproduce the post-peak behaviour in tension [27], we introduce a statistical distribution of the breaking parameters. Gauss and Weibull distributions have been proposed and investigated. [28] showed that the resulting displacement field at the top of the crack is visually the same as long as the distributions are identical in terms of mean value and standard deviation. However, following the conclusions of [22] regarding the crack patterns, a Weibull distribution has been chosen. The Weibull probability density function is:

$$
f(x)=\frac{k}{\lambda}\left(\frac{x}{\lambda}\right)^{k-1} e^{-(x / \lambda)^{k}}
$$

where $\lambda$ is the scale factor and $k$ the shape factor of the Weibull distribution.

We consider that the spatial variability is the same for both breaking parameters. Consequently, the shape factor is kept identical for each parameter and the scale factor alone varies. Moreover, no minimal or maximal value has been assigned to the breaking parameters, contrary to the proposition of [22]. Thus, the number of parameters is minimised.

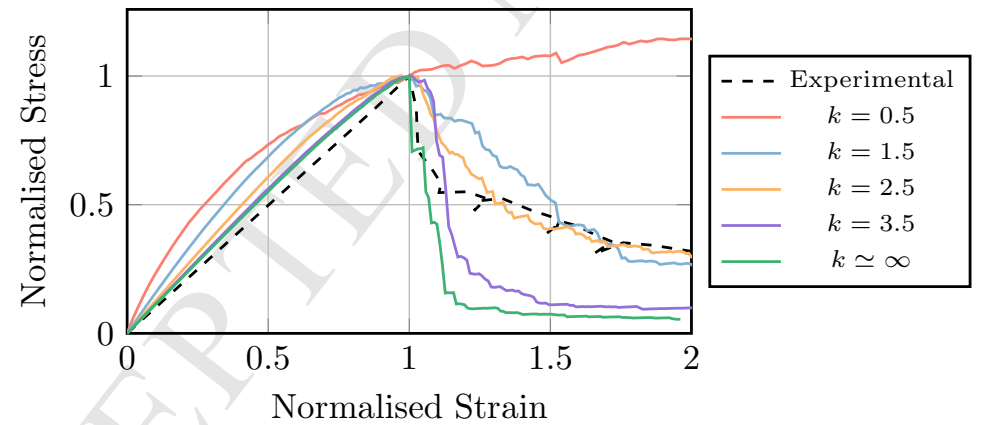

Figure 4: Normalised uni-axial response under tension with respect to the peak stress and strain

On figure 4, we compare the model response in uni-axial tension for a deterministic model - the breaking parameters are the same for every beam $(k \simeq \infty)$ - and a probabilistic model - the breaking parameters follow a spatial variation. We observe a satisfactory softening behaviour in tension if and only if we 
use a probabilistic model. For this comparison, scale factors of the threshold distribution are $\lambda_{\epsilon_{c r}}=2.3910^{-4}$ and $\lambda_{\theta_{c r}}=3.2610^{-3}$.

Further consideration of the spatial variability of the parameters would require the introduction of spatial covariance in the statistical distributions. However, such a process is more complex and is quite similar to building explicit morphological meso-structures [29].

\subsection{Formulation of the local failure criterion}

We have previously proposed to introduce a spatial variability for the breaking thresholds $\epsilon_{i j}^{c r}$ and $\theta_{i j}^{c r}$ in order to dissipate energy in tension. Unfortunately, DEM-based models are also known to display an excessively fragile behaviour in compression [30]. To overcome this limitation, a modified formulation of the failure criterion $P_{i j}\left(\epsilon_{i j} / \epsilon_{i j}^{c r},\left|\theta_{j}-\theta_{i}\right| / \theta_{i j}^{c r}\right)>1$ is proposed to reproduce experimental results [31]. Many formulations of failure criterion can already be found in the literature [24] and some of them offer an accurate description of the complete failure behaviour. [32] tested several formulations based on Rankine's or Mohr-Coulomb's criterion. They observed that the use of extensions and rotations instead of axial forces and bending moments, as originally proposed by [6], does not change the results. Therefore, a criterion based on $\epsilon_{i j}, \theta_{i}$ and $\theta_{j}$ is chosen to remain in the kinematic framework of this beam-particle model.

The simplest failure criterion which correctly captures the tensile failure is a Rankine's criterion based on the beam extension:

$$
P_{i j}^{R, e x t}=\frac{\epsilon_{i j}}{\epsilon_{i j}^{c r}}>1
$$

However, a unique threshold in extension is not sufficient to observe a stiffness loss in compression and it is necessary to add a rotation threshold:

$$
P_{i j}^{R, r o t}=\frac{\left|\theta_{i}-\theta_{j}\right|}{\theta_{i j}^{c r}}>1
$$

Moreover, it is incorrect to separately check $P_{i j}^{R \text {,ext }}>1$ and $P_{i j}^{R, r o t}>1$. Indeed, the failure under bending depends on the state of compression of the beam. Thus, we combine the two criteria: 


$$
P_{i j}^{M C}=\frac{\epsilon_{i j}}{\epsilon_{i j}^{c r}}+\frac{\left|\theta_{i}-\theta_{j}\right|}{\theta_{i j}^{c r}}>1
$$

One should note that the extension term is not squared as proposed by [4]. Indeed, such a choice would not only cause failure under pure contraction but would also cancel the strengthening of the beam in compression.

The formulation can be seen as a Mohr-Coulomb-type criterion (see figure 5) controlled with only two parameters, which set for each beam the cone vertex position $\epsilon_{i j}^{c r}$ and the intersection of its edge with the zero-extension plane $\theta_{i j}^{c r}$. The resistance in rotation increases with the level of contraction inside the beam. Such a formulation of the failure criterion can be seen as an homogenisation of lower scale degradation mechanisms of voids and defects [33].

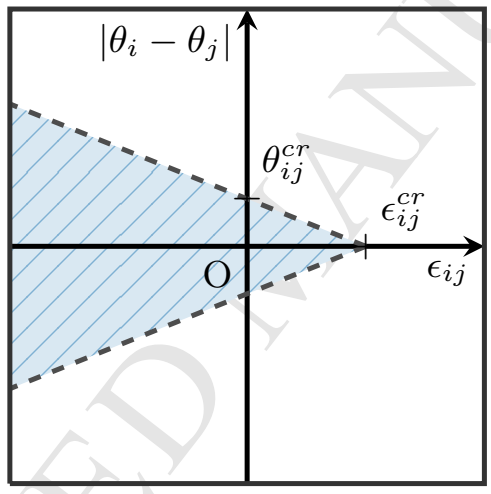

Figure 5: Mohr-Coulomb failure criterion

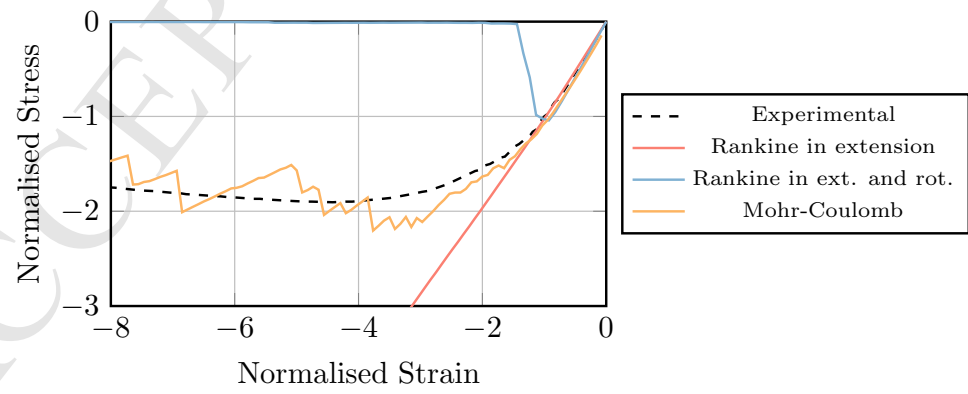

Figure 6: Normalised uni-axial response under compression with respect to the elastic limit stress and strain 
The model response under uni-axial compression (see figure 6) shows the necessity of using a Mohr-Coulomb criterion to capture the ductile failure in compression. For this comparison, the scale factors of the threshold distribution are $\lambda_{\epsilon_{c r}}=2.3910^{-4}$ and $\lambda_{\theta_{c r}}=3.2610^{-3}$ (when used) and $k=2.3$.

\section{Parameter Identification}

\subsection{Particle size}

The density of the mesh is set by setting the average size of the particles $l_{p}$. A compromise must be found in order to limit the computation time while ensuring the macroscopic homogeneity of the material.

On one hand, [10] showed that there should be at least 50 particles in the smallest dimension of the specimen in order to obtain the convergence of the elastic properties. One should note that the convergence of the failure properties - such as the peak load or the amount of dissipated energy - cannot be achieved with this kind of model [34]. However, an increase in the number of particles still leads to a decrease of the dispersion of all the properties - be they elastic or failure properties.

On the other hand, the total number of particles should be kept inferior to 50000 if one wants to obtain results within reasonable computation time and use of memory on a regular computer.

Finally, one should note that the dependency of the failure properties on the density of the mesh means that the calibration of the full set of parameters is relevant only if one does not change $l_{p}$.

\subsection{Elasticity}

The procedure to identify the two elastic parameters - namely the beams' Young's modulus $E$ and their inertia coefficient alpha - has been proposed by [35]. These two parameters are calibrated in order to fit the elastic material properties $E_{m}$, the Young's modulus, and $\nu_{m}$, the Poisson coefficient. These material properties are obtained thanks to a uni-axial tensile test. The sensibility analysis showed that the material's Young's modulus $E_{m}$ is proportional to 
the beam's Young's modulus $E$ and that the material's Poisson coefficient $\nu_{m}$ does not depend on the beam's Young's modulus E. Therefore the two steps of the procedure are:

- Calibrate $\alpha$ with respect to material's Poisson coefficient $\nu_{m}$.

- Calibrate $E$ with respect to material's Young's coefficient $E_{m}$.

\subsection{Failure}

The calibration of the failure behaviour is achieved by choosing the breaking parameters. Given that we chose a Weibull distribution and a Mohr-Coulombtype failure criterion, those parameters are the shared shape factor $k$ and the scale factors of the Weibull distributions of the critical strain, $\lambda_{\epsilon_{c r}}$, and of the critical rotation $\lambda_{\theta_{c r}}$.

We observed that the peak load of failure tests which show beams breaking in bending is influenced by $k, \lambda_{\epsilon_{c r}}$ and $\lambda_{\theta_{c r}}$. Moreover, the peak load of failure tests which show beams breaking in extension is impacted by $k$ and $\lambda_{\epsilon_{c r}}$. Finally, the post-peak phase of a failure test in extension is only affected by $k$.

The following steps are adopted:

- Calibrate $k$ on the post-peak phase of a complete failure test in extension,

- Calibrate $\lambda_{\epsilon_{c r}}$ on the peak load of a failure test involving extension breaking such as the direct tension test, the three-point bending test or the wedge splitting test,

- Calibrate $\lambda_{\theta_{c r}}$ on the peak load of a failure test involving bending breaking such as the compression test or the Brazilian splitting test.

As randomness is introduced through the mesh generation and the fracture parameters' distribution, an average numerical response of 50 computations on the same specimen is used to fit the experimental response.

One should note that the splitting test, the compression test and the threepoint bending test are standardized tests for experimentally characterising a 
specific concrete and should therefore be easily accessible. However, the splitting test should be preferred to the compression test. Indeed, the friction effects at the loading surfaces significantly impact the failure behaviour and are reproduced through the loading conditions with difficulty.

Finally, the failure parameters can be calibrated on the experimental tensile strength, compression strength and fracture energy. This identification is however limited because the experimental boundary conditions for the determination of the compression strength are unknown and the fracture energy extracted from a three-point bending test gives less information than the complete post-peak behaviour of this test.

\subsection{Friction}

Finally, the calibration of the friction coefficient $\mu$-introduced in the formulae of the frictional force of the contact mechanism obeying a Coulomb criterion - is carried out.

Here again, an inverse identification can be used to calibrate the friction coefficient. Indeed, [36] show that the confined compression test response is impacted by the value of $\mu$. The simulation with the beam-particle model of a compression test under a lateral confinement pressure of $10 \mathrm{MPa}$ shows an increase of the post-peak energy dissipation with an increase of the friction coefficient (see on figure 7).

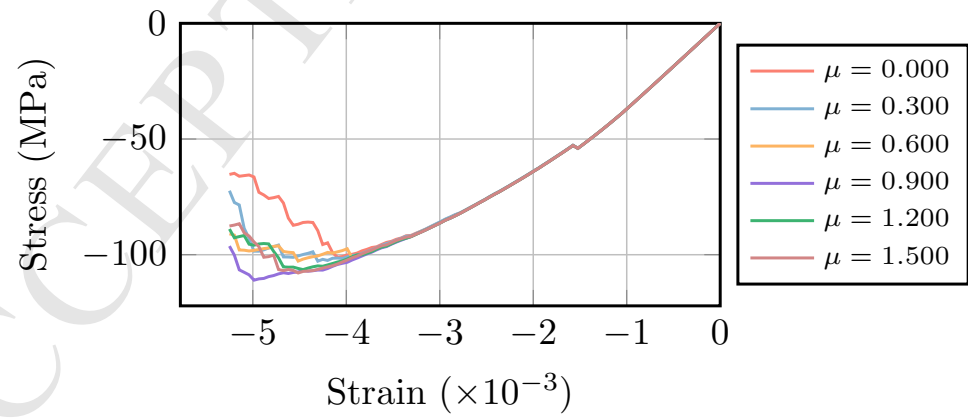

Figure 7: Influence of the friction coefficient $\mu$ on the compression response for a given confinement pressure: $10 \mathrm{MPa}$

However, the difficulty in reproducing the loading conditions of such a test 
prevents us from using it to conduct an accurate identification of the friction coefficient. For this reason, this coefficient is set to an average value $\mu=0.7$, which leads to a coherent ductility. Such a value is found commonly in the literature [37].

\section{Illustration of the identification process}

In section 4 , multiple tests were introduced to perform the identification process. Obviously, those tests must be carried out on the same batch of concrete - or at least the same formulation - as the experiment of interest.

In this section, the experimental data from [38] is used. It contains results from various three-point bending tests (see set-up on figure 8), splitting tests, and compression tests.

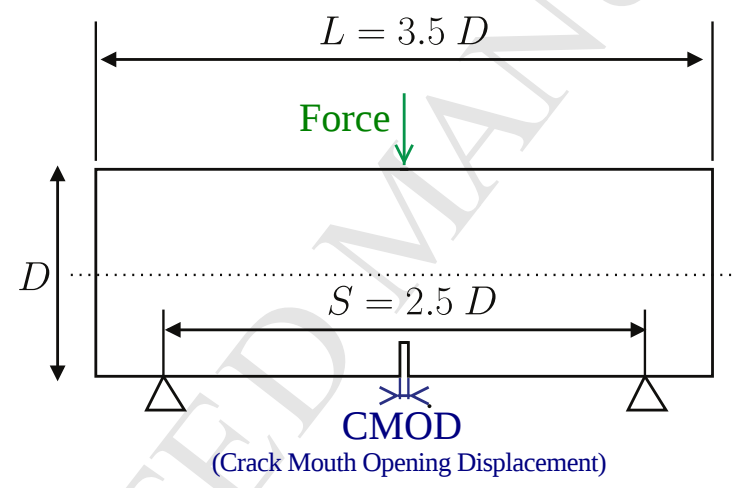

Figure 8: Description of the 3-point bending test's set-up [38]

\subsection{Calibration}

The particle size is arbitrarily set at $l_{p}=2 \mathrm{~mm}$. This size ensures a correct density regarding isotropy for the majority of the tests. Nonetheless, the smallest beam might show a bias as there will only be 25 particles along the smallest dimension of the beam.

In order to reproduce the elastic properties of the concrete, $E_{m}=37 \mathrm{GPa}$ and $\nu_{m}=0.21$, the beam's Young's modulus is fixed to $E=46 \mathrm{GPa}$ and the coefficient of inertia to $\alpha=0.83$. 
Before calibrating the failure parameters $k, \lambda_{\epsilon_{c r}}$ and $\lambda_{\theta_{c r}}$, we show their influence on the failure behaviour of a 3-point bending test and a splitting test through a sensibility analysis.

First, we observe on figure 9 the influence of the three parameters on the post-peak behaviour of a 3-point bending test on a un-notched beam of length $0.35 \mathrm{~m}$ and height $0.1 \mathrm{~m}$. On this figure, the normalised behaviour is plotted - i.e. $F / F_{\max }$ versus $C M O D / C M O D_{\max }$. This way, the impact of the parameters on the post-peak phase is not perturbed by their influence on the peak. We can observe that the shape parameter $k$ alone influences the post-peak behaviour. Its value is then identified to $k=2.8$.

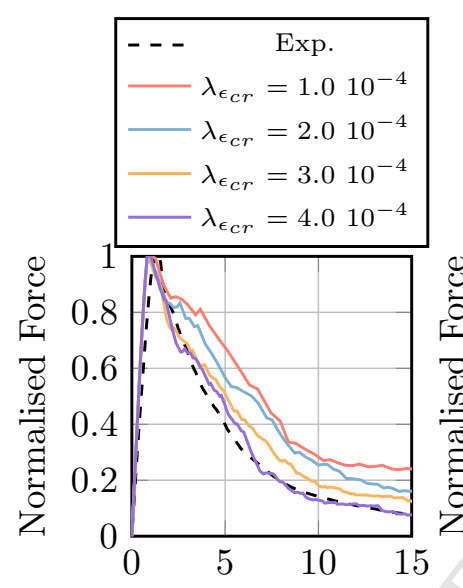

Normalised CMOD

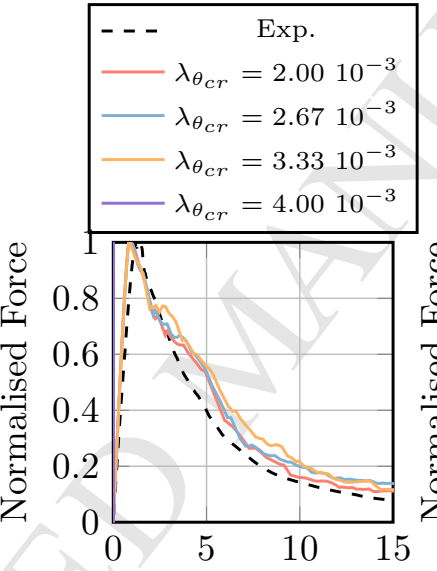

Normalised CMOD

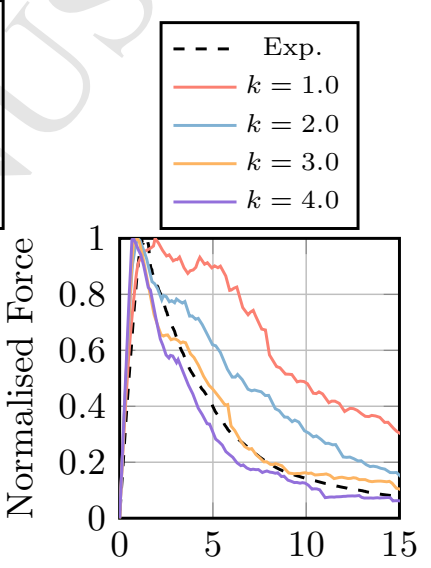

Normalised CMOD

Figure 9: Influence of the failure parameters on the 3-point bending test's normalised response: $\lambda_{\epsilon_{c r}}$ (left), $\lambda_{\theta_{c r}}$ (center), and $k$ (right) 

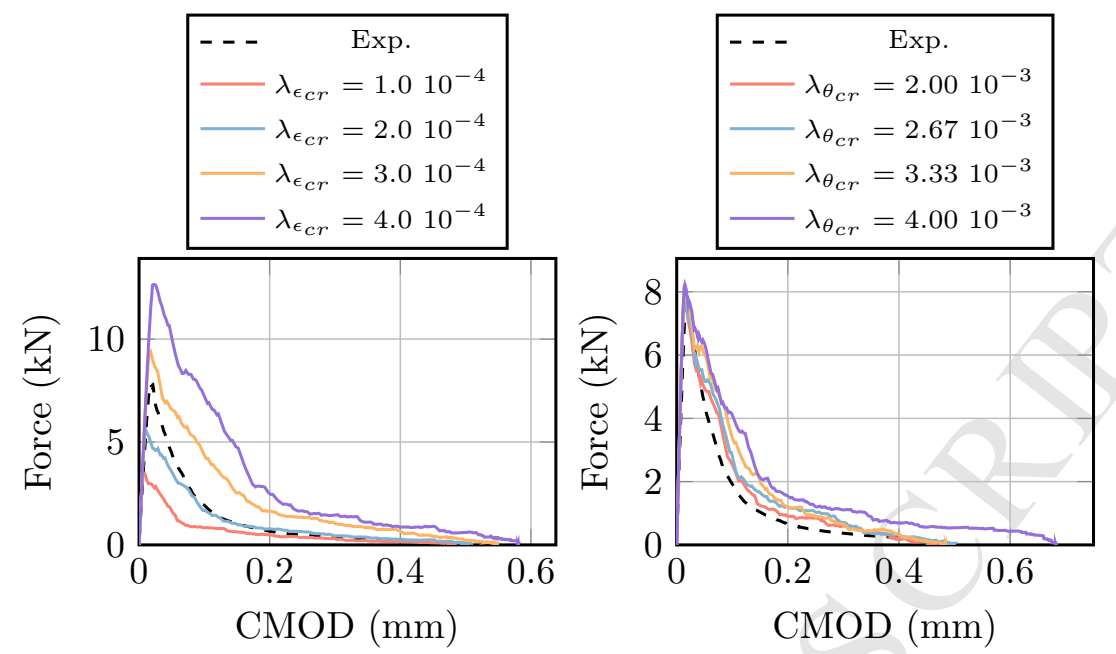

Figure 10: Influence of the failure parameters on the 3-point bending test's response: $\lambda_{\epsilon_{c r}}$ (left), and $\lambda_{\theta_{c r}}$ (right)

Then, the response of the same 3-point bending test is used to analyse the influence of the two scale parameters on the peak-load (see figure 10). Mode I failure is mostly involved, thus $\lambda_{\theta_{c r}}$ does not impact the peak-load. The value of the critical strain scale factor is identified to $\lambda_{\epsilon_{c r}}=2.3910^{-4}$.

Finally, the response of the splitting test on a cylinder of diameter 0.215 $\mathrm{m}$, and particularly the peak-load value $f_{t}=3.9 \mathrm{MPa}$, is used to identify the critical rotation scale factor $\lambda_{\theta_{c r}}=3.2610^{-3}$ (see figure 11). The equivalent stress is computed as $\tilde{\sigma}=2 F / \pi D L$, with $F$ the applied force, $D$ the diameter of the specimen and $L$ its length, here equals to $1 \mathrm{~m}$ due to bi-dimensional computations. 


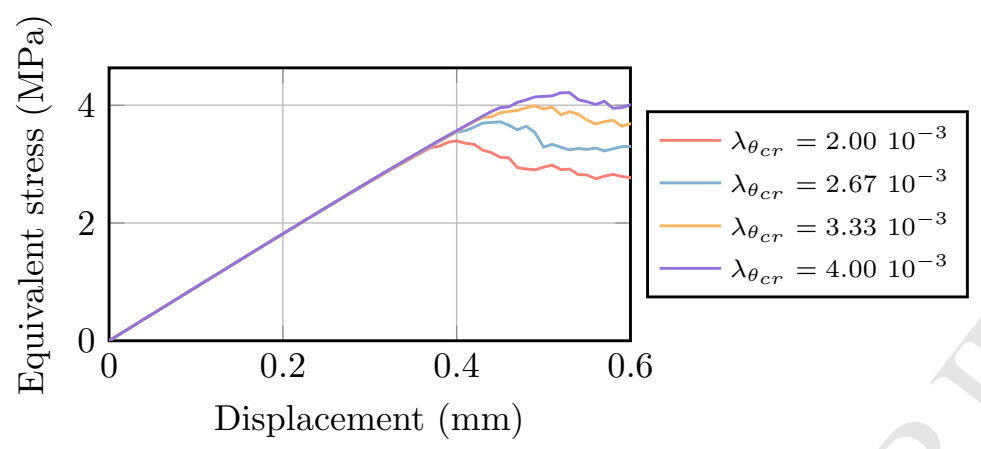

Figure 11: Influence of the failure parameters on the splitting test's response: $\lambda_{\theta_{c r}}$

The set of parameters for this batch of concrete is recapped in table 1 .

\begin{tabular}{|c|c|c|c|c|c|c|}
$l_{p}(\mathrm{~m})$ & $\alpha$ & $E(\mathrm{GPa})$ & $\lambda_{\epsilon_{c r}}$ & $\lambda_{\theta_{c r}}$ & $k$ & $\mu$ \\
\hline 0.002 & 0.83 & 46 & $2.3910^{-4}$ & $3.2610^{-3}$ & 2.8 & 0.7
\end{tabular}

Table 1: Parameters values for the tests simulations

\subsection{Validation of the identification process}

The set of parameters obtained from a 3-point bending test on a half-notched beam of length $0.35 \mathrm{~m}$ and height $0.1 \mathrm{~m}$ and a splitting test on a cylinder of radius $0.215 \mathrm{~m}$ are applied to the study of 3-point bending test on beams of different sizes and various notches and to the study of a compression test.

\subsubsection{Size and shape effects on a 3-point bending test}

The reproduction of the size and shape effects is crucial when dealing with the modelling of failure in concrete. In general, a correct reproduction of those effects on the peak-load and of the post-peak behaviour requires calibrating the model on several sizes (see [39] for non-local damage models or [40] for cohesive crack models). Furthermore, the sets of parameters obtained are generally limited to a small range of sizes and geometries [38]. Finally, the reproduction of the size effect for the non-local damage models implies the calibration of the internal length.

Here, our internal length is a mesh density measure that is freely chosen with regard to the size and shape effects at least. Then, the model - which is calibrated on a unique size and a unique notch length - gives predictive results 
for three sizes and two notch lengths (see figures 12 and 13). The beam-particle model is able to reproduce the size effect not only on the nominal strength but also on the slope of the post-peak phase.

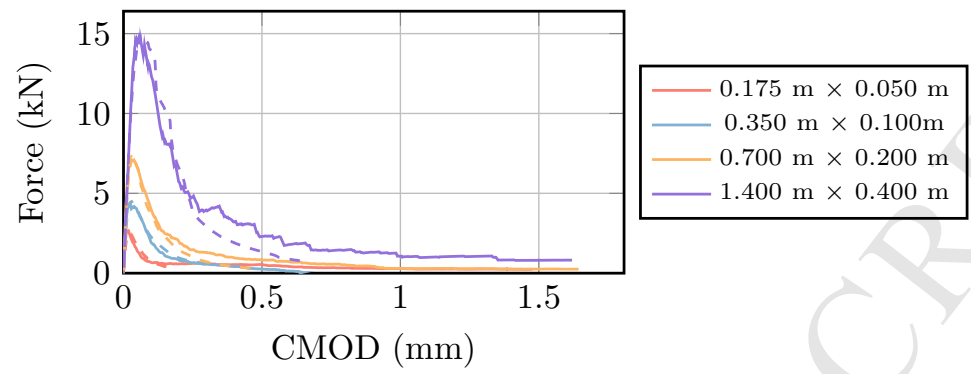

Figure 12: Influence of the specimen size on the 3-point bending test's response of the fifthnotched beams: experiments (dashed), simulations (plain)

In order to correctly reproduce the size effect and the shape effect, one must consider the true nature of cracking, which is not localised in a sharp crack tip, but is rather spread over a certain volume called fracture process zone [41]. Looking at the crack patterns obtained for different notch lengths (see figure 14), we conclude that the beam-particle model ensures a correct representation of the crack initiation and propagation.

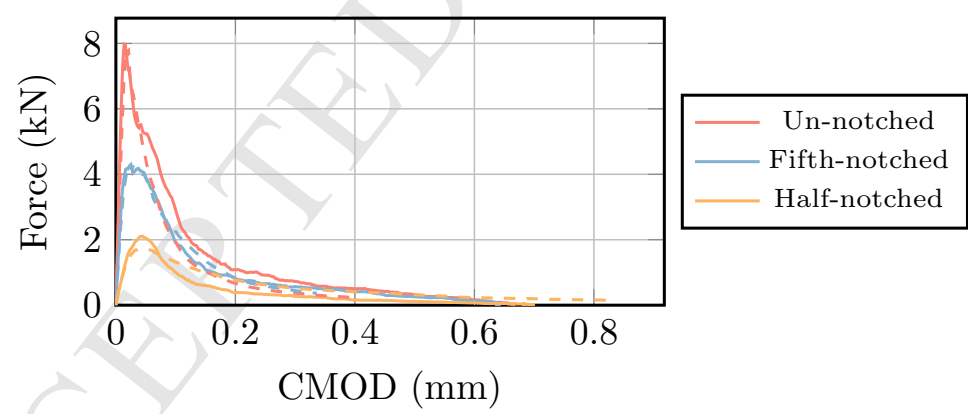

Figure 13: Influence of the notch depth on the 3-point bending test's response on one of the small $(0.350 \mathrm{~m} \times 0.100 \mathrm{~m})$ beams: experiments (dashed), simulations (plain) 

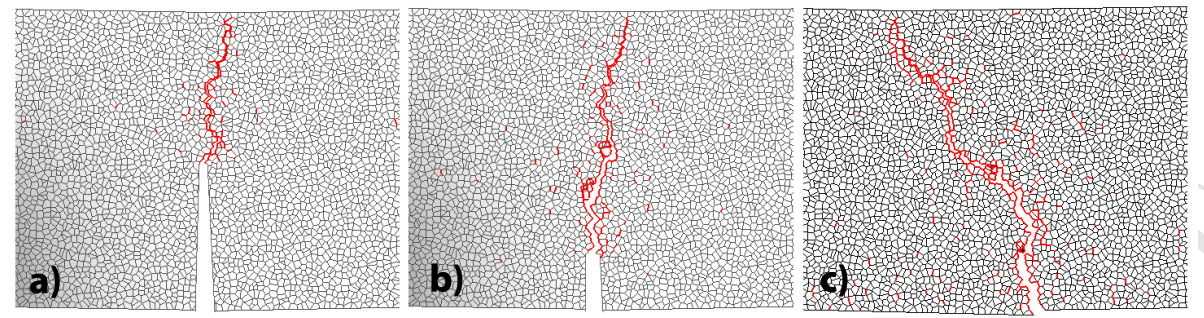

Figure 14: Influence of the notch-length on the crack pattern (focus on the central part of the beam): a) half-notched beam, b) fifth-notched beam and c) un-notched beam

\subsubsection{Dispersion of the results}

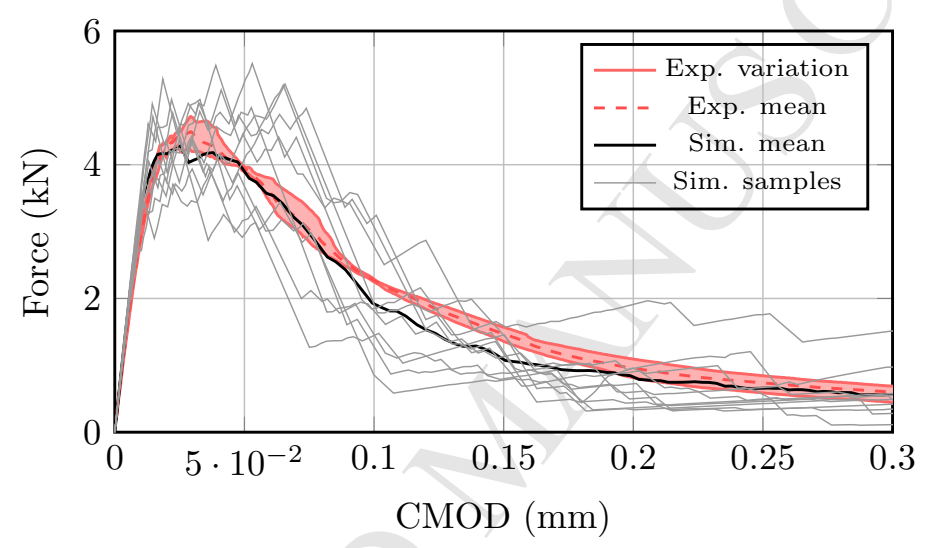

Figure 15: Variability of the response of a small fifth-notched 3-point bending test

The dispersion of the model's responses is studied on a 3-point bending test on a small $(0.350 \mathrm{~m} \times 0.100 \mathrm{~m})$ fifth-notched beam (see figure 15). Several hypotheses can explain the fact that the variability of the simulated responses is higher than the experimental ones. First, only three experiments were performed for each size of beam which might not be enough to observe the full dispersion of the experimental results. Second, the shape parameters of the thresholds distributions $k$ influences not only the post-peak behaviour but also the dispersion of the results. Last, the mesh is not refined enough for this size of beam, leading to a cracking-pattern excessively influenced by particles shape and spatial organization.

Because we do not have access to more than three experimental results, and 
because we do not want to change the shape factor $k$, we investigate the influence of the number of particles on the dispersion of the results. The number of particles increases with the size of the specimen and its influence on the standard deviation is given on figure 16. First, we observe that the experimental relative standard deviation is not constant which is due to the lack of experimental results. Then, we note that the numerical relative standard deviation decreases with the number of particles and seems to converge toward the experimental value. Therefore, the setting of the particle length should not only ensure the convergence of the mean elastic properties but also the convergence of the relative standard deviation of the peak.

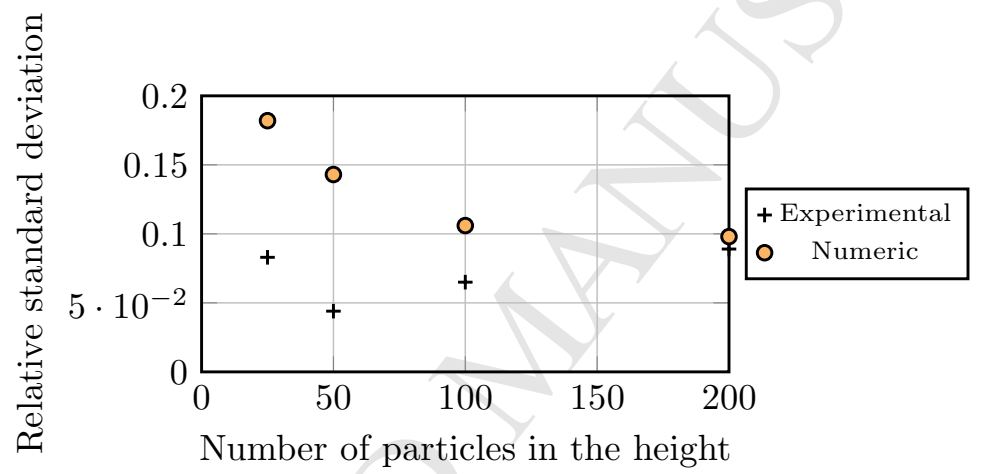

Figure 16: Influence of the number of particles in the height on the dispersion of the peak load

Nevertheless, using a coarse mesh remains an interesting option to obtain conservative results - the experimental range of responses being entirely included in the numerical one - for a limited computational cost.

\subsubsection{Compression response}

Compression tests are intricate because solicitations near the loading platens are difficult to simulate. Indeed, highly multi-axial stress states can occur depending on the adherence between the specimen and the platens. Due to the quasi-static framework, boundary conditions at the platens have to be applied in a specific manner to avoid rigid body motion without adding unrealistic constraints. 
Application of partially restrained boundary conditions. Figure 17 illustrates the application of partially restrained boundary conditions.

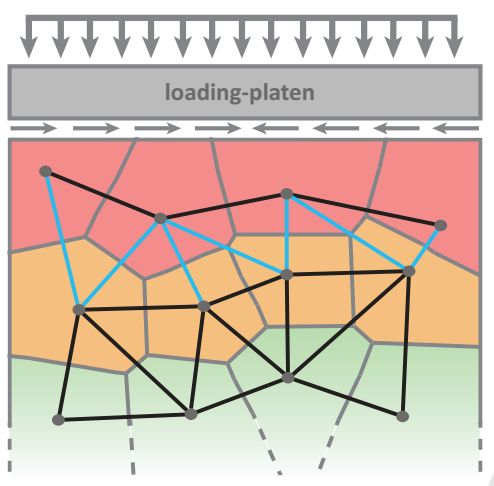

Figure 17: Schematic description of the application of partially restrained boundary conditions

The loading platen interacts with the first layer of particles. To simulate a perfectly sliding interface between the specimen and the platens, we should apply vertical displacements on the first layer (in red) of particles, without applying any horizontal displacement. Unfortunately, rigid-body motion will occur in the horizontal direction. A possible solution would be to lock a red particle horizontally. However, failure can localise around this particle and brings back the original issue of rigid-body motion. Finally, if failure is prevented, horizontal constraints appear and bias the results.

The solution proposed here consists in applying boundary conditions on two layers of particles instead. On the first layer vertical displacements are applied and the horizontal displacements are completely locked. On the second layer (in orange), only the vertical displacement is imposed. At this point, boundary conditions are equivalent to perfectly adherent loading platens.

In order to simulate the influence of partial adherence, the amount of stress transferred from the loading platen to the specimen must be adjusted. To do this, the stiffness of the beams linking the first layer to the second layer is reduced by a factor $k_{p}$. These beams are also considered unbreakable to avoid localisation of failure due to their higher compliance. A null stiffness $\left(k_{p}=0\right)$ is equivalent to having perfect sliding in between the loading platen and the 
specimen, while an intact stiffness $\left(k_{p}=1\right)$, as aforementioned, is equivalent to having perfect adherence in between the platens and the specimen.

Simulations. The numerical results for a compression test on a cylinder of height $0.14 \mathrm{~m}$ and diameter $0.074 \mathrm{~m}$ are compared to experimental ones on figure 18 . The sensibility of the compression results on the adherence level is studied. Because teflon was placed between the loading platens and the specimen, one could have expected to observe no adherence $\left(k_{p}=0\right)$. However, the experimental response is accurately reproduced for softened boundary conditions with $k_{p}=0.0015$.

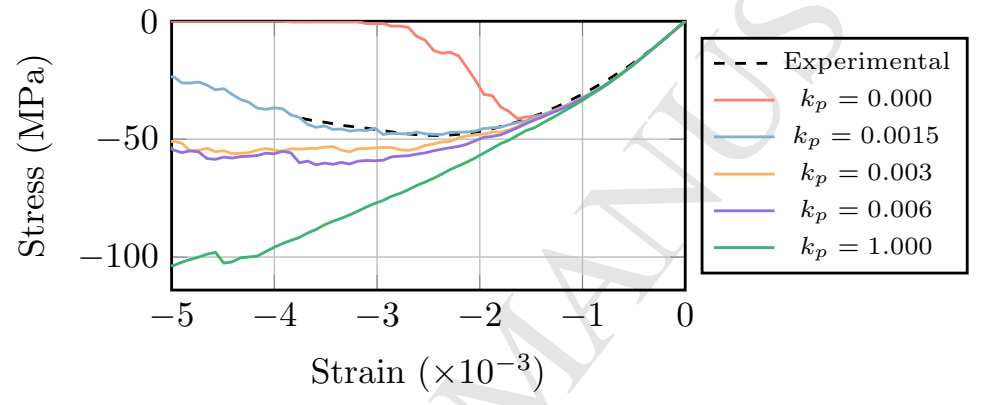

Figure 18: Influence of the boundary conditions on the response of a compression test

Experimentally [42], the inclination of the localised macroscopic crack has been observed to depend on the adherence level of the platens. The beamparticle model with the modification to partially apply restrained boundary conditions allows the reproduction of the experimental results: the crack pattern slope increases with the friction (see dashed red line on figure 19). 

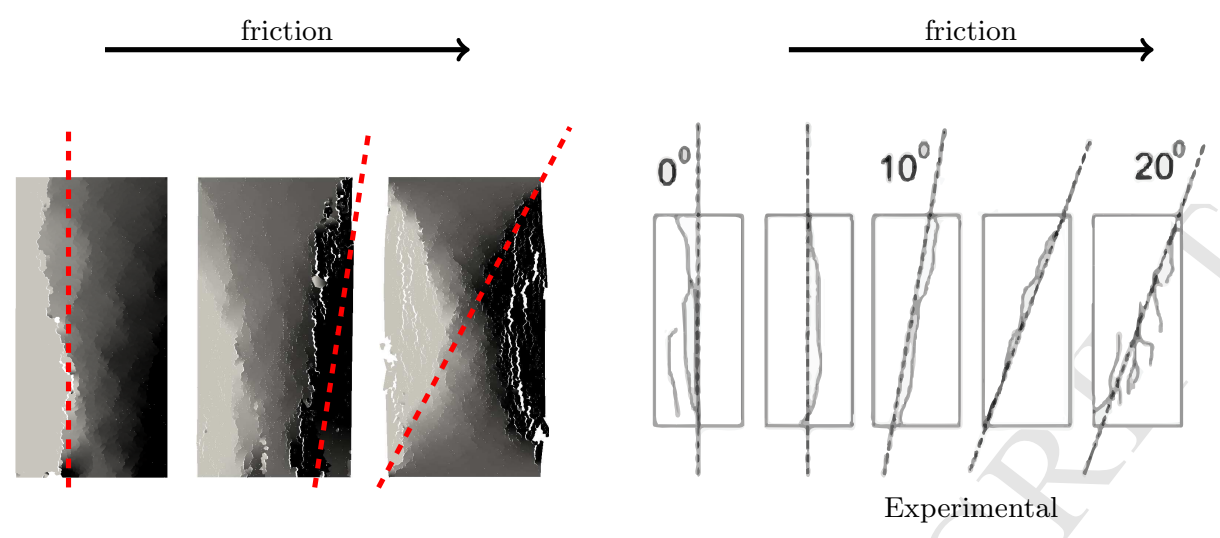

Figure 19: Influence of the adherence at the loaded ends on the cracking patterns in compression

\section{Complex loading and failure}

The validation of the model is continued with quasi-structural test cases. Mode I failure has been previously studied, therefore only mixed mode (I/II) failure and mode II failure test cases are investigated. The model parameters previously calibrated cannot be directly used for those test cases. Unfortunately, the available experimental results do not allow us to rerun a complete identification process. Therefore, the beams' Young's modulus $E$ and the scale factors of their failure parameters' distributions $\left(\lambda_{\epsilon_{c r}}, \lambda_{\theta_{c r}}\right)$ are slightly adjusted on one of the provided structural responses. For this reason, the results presented in this section cannot be considered as entirely predictive. Nevertheless, it is proven that the beam-particle model is able to predict the impact of a change of geometry or boundary conditions on the load-displacement responses and the cracking patterns.

\subsection{Mixed mode I/II failure: Schlangen's beams tests}

[43] tested single-edge (SEN) and double-edge (DEN) notched beams under four-point bending (see the experimental set-up on figure 20). The evolution of the applied force, of the crack-mouth opening (CMOD) and of the crack-mouth sliding (CMSD) displacements are given for sliding and fixed supports. 


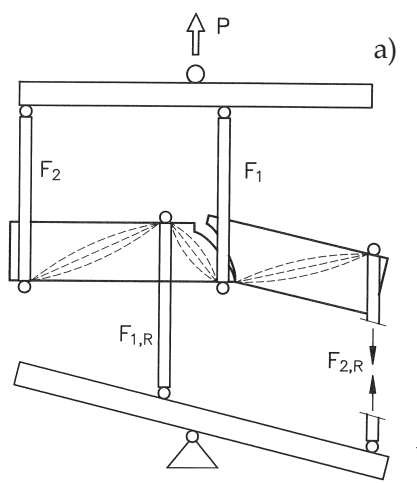

a)

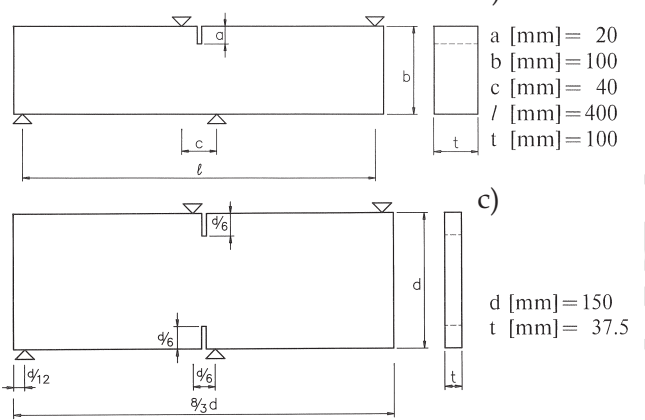

Figure 20: Schlangen's beams tests: the set-up (a) and dimensions of single-edge (b) and double-edge (c) notched beams

The model is slightly re-calibrated on the force versus CMOD response of the SEN beam test with sliding supports. The beams' Young's modulus $E$ is set to fit the initial slope of the response. The critical extension scale factor $\lambda_{\epsilon_{c r}}$ is set to obtain the most accurate fit of the peak-load value and the critical rotation scale factor $\lambda_{\theta_{c r}}$ is set to reproduce the post-peak behaviour. The set of parameters is recapped in table 2 .

\begin{tabular}{|c|c|c|c|c|c|c|}
$l_{p}(\mathrm{~m})$ & $\alpha$ & $E(\mathrm{GPa})$ & $\lambda_{\epsilon_{c r}}$ & $\lambda_{\theta_{c r}}$ & $k$ & $\mu$ \\
\hline 0.002 & 0.83 & 46 & $3.2610^{-4}$ & $3.9110^{-3}$ & 2.8 & 0.7
\end{tabular}

Table 2: Parameters values for Schlangen's beams tests simulations

\subsubsection{Sliding supports}

First, we focus on the responses for SEN and DEN beams with sliding supports. Three of the four supports have free horizontal displacement boundary conditions, while the bottom left corner support is locked in order to avoid rigid body motion.

The model is able to correctly reproduce the experimental evolutions of the force versus CMOD and of the force versus CMSD of both SEN and DEN beams (see figure 21). 


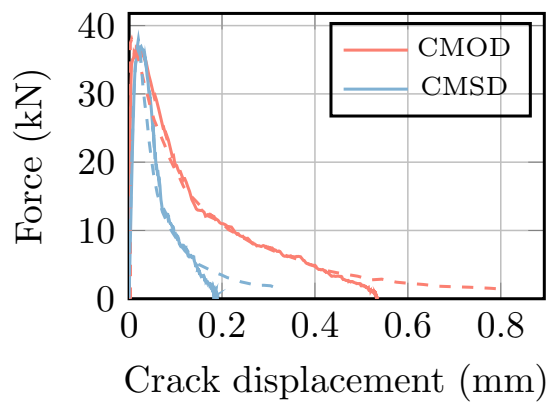

(a) single-edge notched beam

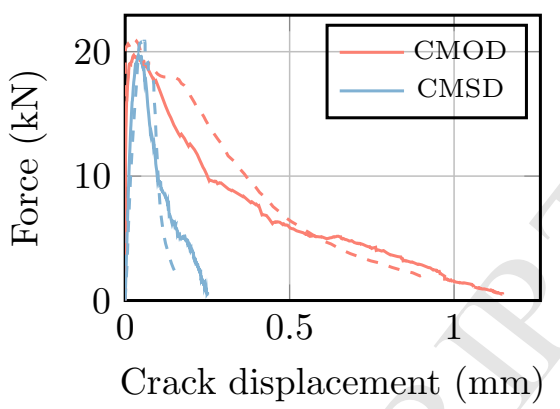

(b) double-edge notched beam

Figure 21: Responses of the model for Schlangen tests with sliding supports: experiments (dashed), simulations (plain)

The numerical crack patterns are also in agreement with the experimental ones (see figure 22).

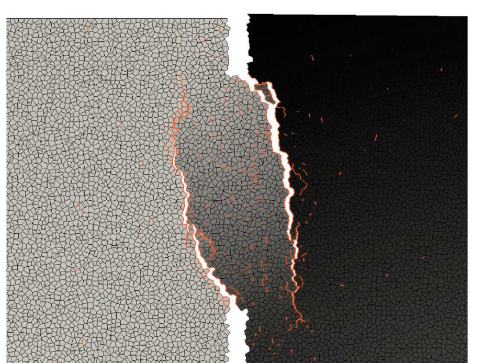

a)

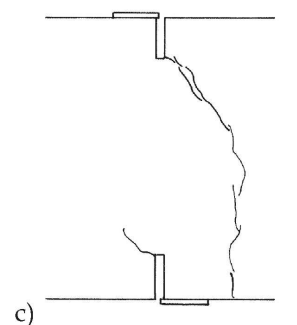

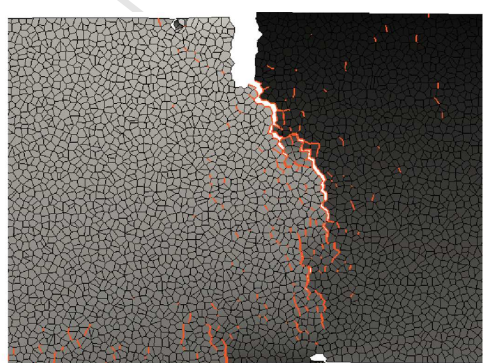

b)

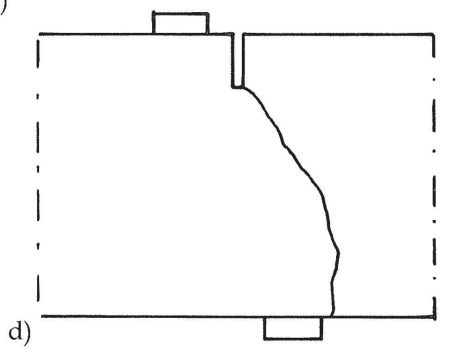

Figure 22: Comparison between the numerical crack patterns of the a) SEN beam (respectively b) DEN beam) and the experimental ones of the c) SEN beam (respectively d) DEN beam)

\subsubsection{Fixed supports}

According to finite element analysis of the Schlangen's beams tests performed by [44], the definition of the fixed boundary conditions requires special attention. Indeed, the fixed supports cannot be considered perfectly stiff. Therefore, 
the methodology presented in 5.2.3 has been applied to soften the boundary conditions. The stiffness reduction coefficient varies to model boundary conditions from perfectly fixed supports $\left(k_{p}=1\right)$ to perfectly sliding supports $\left(k_{p}=0\right)$.

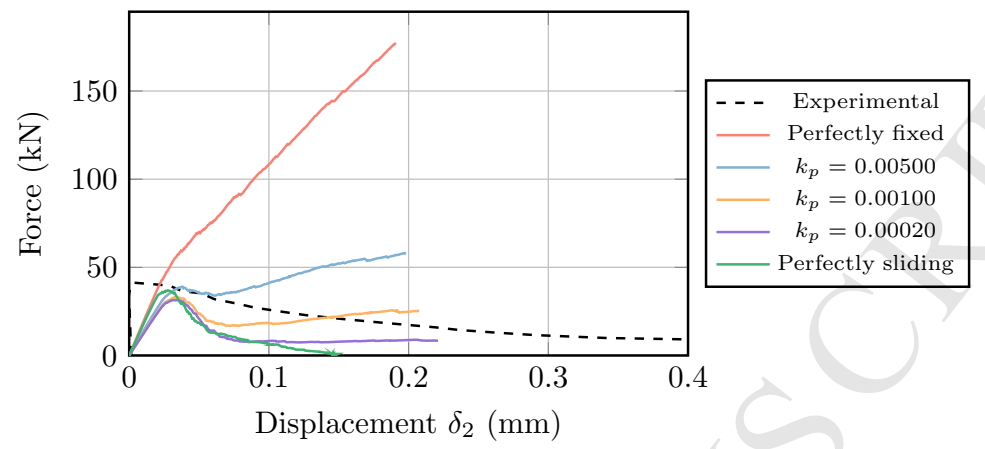

Figure 23: Influence of the compliance of the supports on the model's response for SEN beam tests

For the SEN beam, the vertical displacement $\delta_{2}$ at the right top corner support alone is experimentally available. Unfortunately, this displacement is greatly influenced by rigid body displacements of the experimental loading setup. This is why we were not able to reproduce the experimental evolution of the force versus $\delta_{2}$ (see on figure 23).

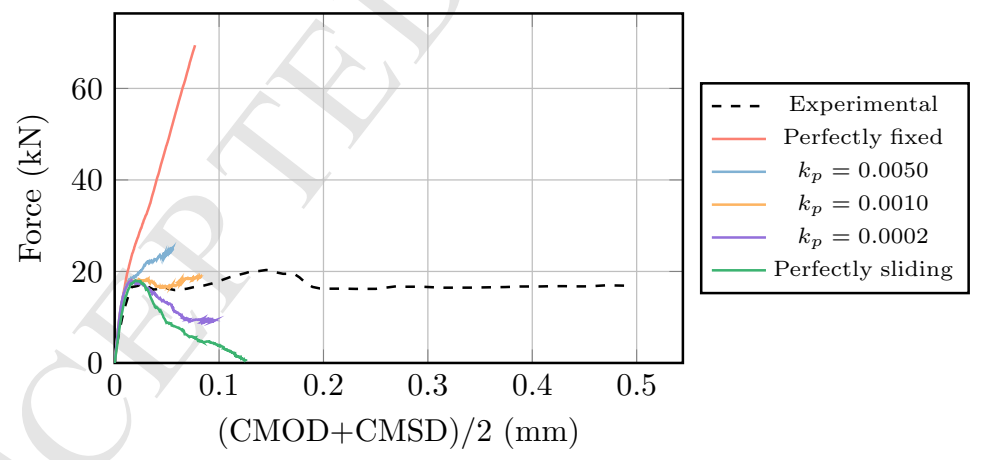

Figure 24: Response of the model for DEN beam tests with corrected friction at the supports

For the DEN beam, an experimental measure of the average of the CMOD and the CMSD is available. We were able to compute the evolution of the force with this measure. The comparison with the experimental results is performed 
on figure 24. For perfectly fixed supports, the core cannot expand horizontally and so the cracks are prevented from opening and propagating. As a result, the peak-load is never reached. Accurate results in terms of response are found for a stiffness reduction coefficient of $k_{p}=0.001$.

Regarding crack propagation (see figure 25 and 26), the patterns are inaccurate for both the SEN beam and the DEN beam if the supports are fixed. Indeed, confinement is generated in the mid-section and the cracks cannot propagate. If the stiffness of the support is sufficiently reduced $\left(k_{p}=0.001\right)$, the experimental crack patterns can be reproduced. Indeed, for the SEN beam we reproduce the curved crack between the notch tip and the bottom loading platen as well as the vertical crack under the top loading platen. For the DEN beam, we capture the two antisymmetric curved cracks originating from each notch and a splitting crack in the center of the beam.

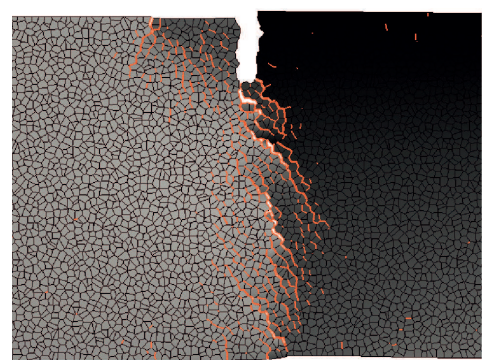

a)

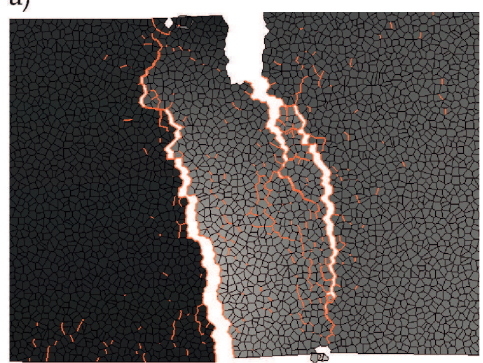

b)

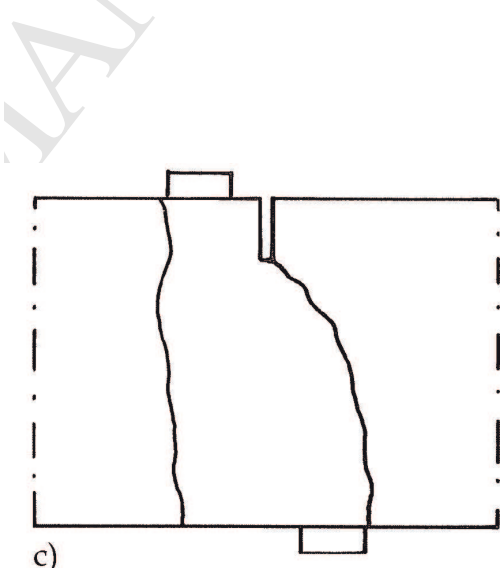

c)

Figure 25: Comparison between the numerical crack patterns with a) fixed supports and b) modified supports and the c) experimental one of the SEN beam 


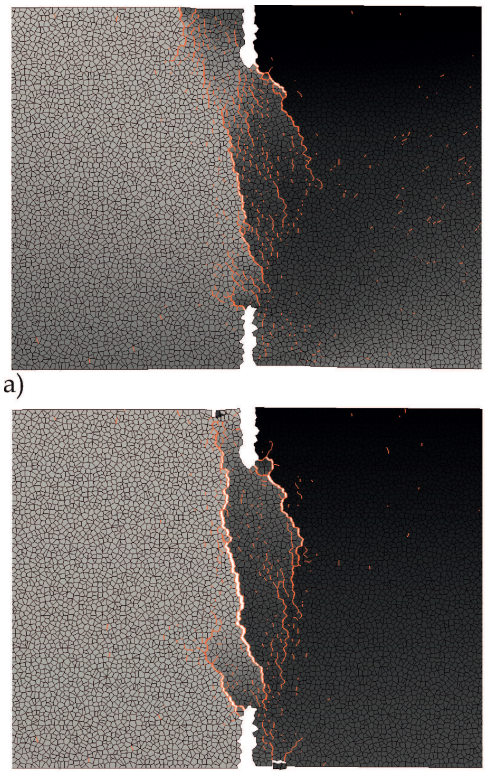

b)

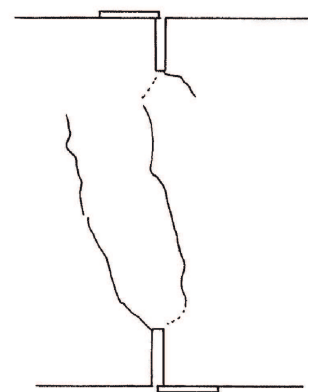

c)

Figure 26: Comparison between the numerical crack patterns with a) fixed supports and b) modified supports and the c) experimental one of the DEN beam

\subsection{Mode II failure: Fenwick and Paulay shear test}

The shear test proposed by [45] allows to test a concrete specimen under pure mode II failure (see figure 27$)$. The specimen (0.45 m long and $0.1 \mathrm{~m}$ high) is first loaded under uni-axial tension to form a localised crack in between two small notches at mid-length of the sample. Then the specimen is maintained to keep the crack opening constant, and shear load is applied.

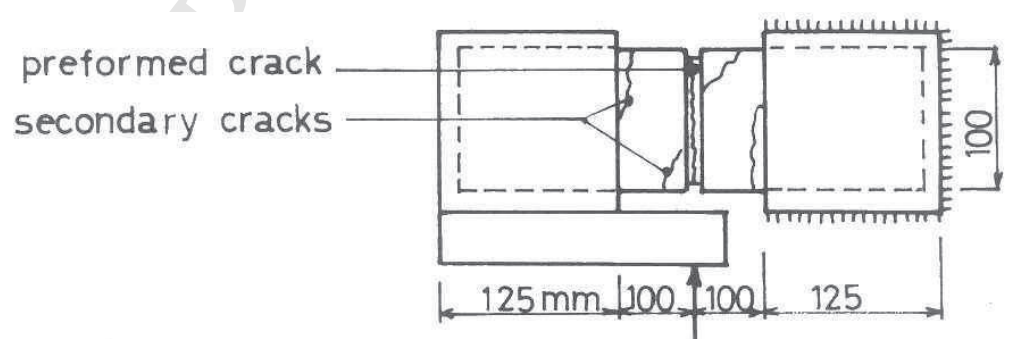

Figure 27: Fenwick and Paulay confined sheared crack test set-up and experimental crack patterns

The evolution of the shear stress with the shear displacement for several crack 
widths - i.e. different confinements levels - is experimentally available [46]. The critical extension scale factor is re-calibrated in order to fit the experimental response for the smallest crack-width $(c w=0.00006 \mathrm{~m})$. The smallest crackwidth is chosen, since it is the closest configuration to a cohesive, unbroken specimen. The whole parameter set is given in table 3 .

\begin{tabular}{|c|c|c|c|c|c|c|}
$l_{p}(\mathrm{~m})$ & $\alpha$ & $E(\mathrm{GPa})$ & $\lambda_{\epsilon_{c r}}$ & $\lambda_{\theta_{c r}}$ & $k$ & $\mu$ \\
\hline 0.002 & 0.83 & 46 & $4.3510^{-4}$ & $3.2610^{-3}$ & 2.8 & 0.7
\end{tabular}

Table 3: Parameters values for Fenwick and Paulay shear tests simulations

The model is able to reproduce not only the peak-load values but also the prepeak overall slope of the smooth experimental curves (see figure 28). Note that the offsets in the pre-peak behaviour (horizontal slope), especially visible at high confinements, are evidence of the "aggregate interlocking" mechanism. Shear stresses transit through the crack because of geometrical incompatibilities, until particles at the crack interface start to spall off, once all their cohesive beams are broken. Sliding is then initiated at the crack lips until new incompatibilities are generated. The model would hardly capture such mechanism without the use of polygonal particles.

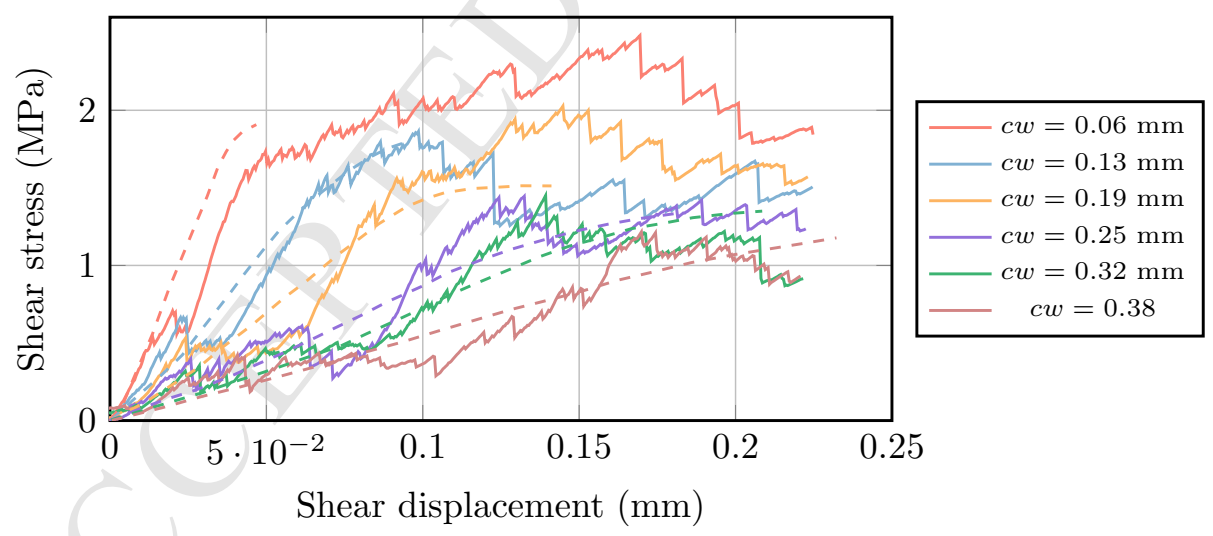

Figure 28: Response of the model for Fenwick and Paulay shear test: experiments (dashed), simulations (plain)

This phenomenon can be observed on figure 30 in the dashed ellipses. A packing of particles, initially linked to the left block, is in contact with the 
other right block enabling the transmission of forces. However contact points in between blocks are sparse, leading to stresses concentrations. The aforementioned packing of particles loses all of its cohesive links with the left block, and therefore spalls off. Thus the displacement restriction of the left block due to the previous contact is removed, and the left block can slide with respect to the right block.

Qualitatively, the numerical crack pattern (see figure 29) is similar to the experimental one (see figure 27). Indeed, we observe the formation of vertical secondary cracks as well as diagonal ones.

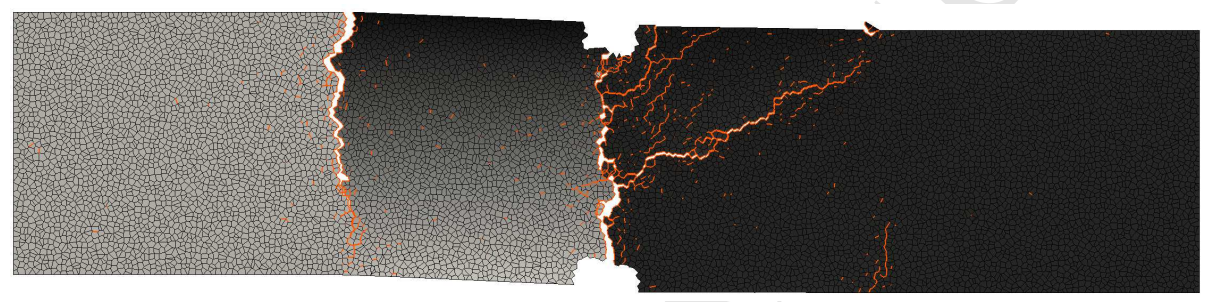

Figure 29: Crack pattern of the model for Fenwick and Paulay shear test at crack-width $c w=0.00032 \mathrm{~mm}$

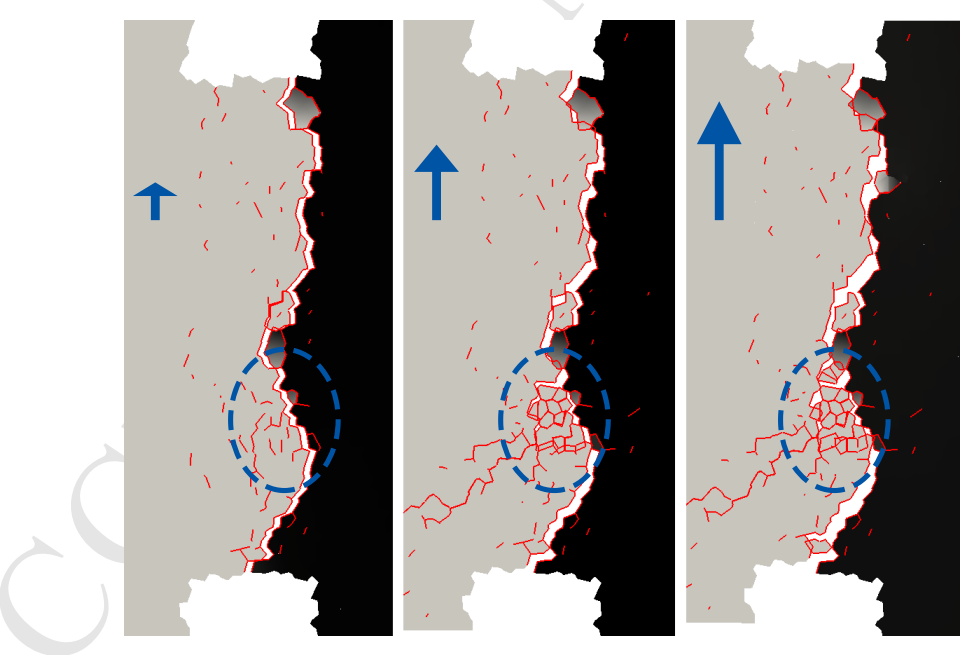

Figure 30: Zoom on the pre-crack during at crack-width $c w=0.00006 \mathrm{~mm}$ for three different applied shear displacements (from left to right): $0.03,0.1$ and $0.2 \mathrm{~mm}$ 


\section{Conclusion}

A beam-particle model has been presented to model the failure behaviour of concrete. Particles are linked by cohesive beams before failure and interact through frictional contact after failure.

This model is based on a lattice representation and hence tends to underestimate the energy dissipation in tension. Spatial variability has therefore been introduced to model a quasi-brittle behaviour in tension. Regarding the ductile behaviour in compression, we showed that using an appropriate failure criterion for the cohesive beams allows us capture it.

The model is governed by seven parameters which have to be correctly identified. In this paper, we proposed a detailed calibration process based on standard tests for concrete characterisation. This procedure is illustrated and a sensibility analysis is performed. We observed that the set of parameters only requires slight adjustments to model different mortars and concrete.

Finally, several test cases were studied to validate the model under compression, mixed mode or pure mode II. For these complex tests, we reproduced the experimental results not only qualitatively - by comparing the crack patterns - but also quantitatively - by comparing the evolution of the load with the displacements. Special attention has to be paid to the boundary conditions and a strategy to apply frictional sliding has been proposed.

To give a better idea of the time efficiency of the model, the required time to compute the cracking pattern of the Schlangen's beam test has been about 10 hours (9 hours and 34 minutes average on the 50 draws of the test at the given mesh refinement) while a finite element model with damage would tackle the problem in minutes. For industrial reinforced concrete structures such as nuclear power plant containment vessels subjected to a complex loading for which hundreds of cracks may initiate and propagate, such numerical procedures would lead to excessive and prohibitive CPU time consumptions. That is why, applications of the model are mostly found in multi-scale modeling methods, which require a mesoscale representation of the cracking of concrete. In this 
case, a good compromise can be obtained between the computation time and the level of precision of the cracking pattern description [34].

In perspective, the extension of the model to 3D studies need to be achieved in order to complete the range of validation tests. The $3 \mathrm{D}$ developments for the lattice part of the model had already been carried out and are relatively straightforward [10]. However, the computation of the contact and friction forces is heavy and therefore prohibited in 3D. Solutions exist to overcome this difficulty. Particles can be approximated by spheres to estimate the intersection [47] or the algorithm can be parallelised.

Future applications of the model could be found in multi-physic problems. It has been shown that beam-particle approaches offer a reliable description of cracking. That being said, it is well known that cracking has multiple influences on the thermo-hydro-mechanical behavior of concrete. For example, the permeability is directly impacted by the presence of macroscopic cracks and information about the opening and orientation of those cracks - which can be easily obtained from a DEM based model - are crucial when computing the anisotropic permeability tensor of a fractured material [48]. Therefore, our approach may allow to model coupled problems such as transfer of water into a cracked material. In fact, some work has been initiated on the study of the permeability of fractured materials such as concrete with the proposed model [49] and further work is ongoing.

\section{References}

[1] C. Oliver-Leblond, B. Richard, A. Delaplace, F. Ragueneau, Cracking analysis of reinforced concrete beams using a finite-discrete element methods approach, FraMCoS 8.

[2] M. Vassaux, B. Richard, F. Ragueneau, Regularised cracks behaviour effects on continuum modelling of quasi-brittle materials under cyclic loading, Engineering Fracture Mechanics. 
[3] A. Hrennikoff, Solution of problems of elasticity by the framework method, Journal of applied mechanics 8 (4) (1941) 169-175.

[4] H. J. Herrmann, A. Hansen, S. Roux, Fracture of disordered, elastic lattices in two dimensions, Physical Review B 39 (1) (1989) 637-648.

[5] Z. P. Bažant, M. Tabbara, M. Kazemi, G. Pijaudier-Cabot, Random particle model for fracture of aggregate and fibre composites, Journal of Engineering Mechanics 116 (8) (1990) 1686-1705.

[6] E. Schlangen, J. G. M. Van Mier, Experimental and numerical analysis of micromechanisms of fracture of cement-based composites, Cement and Concrete Composites 14 (1992) 105-118.

[7] P. A. Cundall, O. D. L. Strack, A discrete numerical model for granular assemblies, Geotechnique 29 (1979) 47-60.

[8] K. Meguro, M. Hakuno, Fracture analyses of concrete structures by the modified distinct element method, Structural Engineering/Earthquake Engineering 6 (2) (1989) 283-294.

[9] G. A. D'Addetta, F. Kun, E. Ramm, On the application of a discrete model to the fracture process of cohesive granular materials, Granular Matter 4 (2002) 77-90.

[10] A. Delaplace, Modélisation discrète appliquée au comportement des matériaux et des structures, Mémoire d'habilitation à diriger des recherches de l'Ecole Normale Supérieure de Cachan.

[11] M. Vassaux, B. Richard, F. Ragueneau, A. Millard, A. Delaplace, Lattice models applied to cyclic behavior description of quasi-brittle materials: advantages of implicit integration, International Journal for Numerical and Analytical Methods in Geomechanics 39 (7) (2015) 775-798.

[12] C. Moukarzel, H. J. Herrmann, A vectorizable random lattice, Journal of Statistical Physics 68 (1992) 911-923. 
[13] E. Schlangen, E. J. Garbozi, Fracture simulations of concrete using lattice models : computational aspects, Eng. Fracture Mech. 57 (1997) 319-332.

[14] J. G. M. Van Mier, M. R. A. Van Vliet, Influence of microstructure of concrete on size/scale effects in tensile fracture, Engineering Fracture Mechanics 70 (2003) 2281-2306.

[15] E. Perkins, J. R. Williams, A fast contact detection algorithm insensitive to object sizes, Engineering Computations 18 (1/2) (2001) 48-62.

[16] J. O'Rourke, C.-B. Chien, T. Olson, D. Naddor, A new linear algorithm for intersecting convex polygons, Computer Graphics and Image Processing 19 (4) (1982) 384-391.

[17] H.-J. Tillemans, H. J. Herrmann, Simulating deformations of granular solids under shear, Physica A: Statistical Mechanics and its Applications 217 (3-4) (1995) 261-288.

[18] H. Reinhardt, J. Weerheijm, Tensile fracture of concrete at high loading rates taking account of inertia and crack velocity effects, International Journal of Fracture 51 (1) (1991) 31-42.

[19] J. G. Rots, S. Invernizzi, B. Belletti, Saw-tooth softening/stiffening - a stable computational procedure for rc structures, Computers \& Concrete 3 (2006) 213-233.

[20] G. Cusatis, Z. P. Bažant, L. Cedolin, Confinement-shear lattice model for concrete damage in tension and compression. i: Theory, Journal of Engineering Mechanics 129 (12) (2003) 1439-1448.

[21] G. Cusatis, Z. Bažant, L. Cedolin, Confinement-shear lattice csl model for fracture propagation in concrete, Computational Methods Applied Mechanical Engineering 195 (52) (2006) 7154-7171.

[22] J. G. M. Van Mier, M. R. A. Van Vliet, T. K. Wang, Fracture mechanisms in particle composites: statistical aspects in lattice type analysis, Mech. Mater 34 (2002) 705-724. 
[23] P. Grassl, M. Jirásek, Meso-scale approach to modelling the fracture process zone of concrete subjected to uniaxial tension, International Journal of Solids and Structures 47 (2010) 957-968.

[24] C. T. Davie, N. Bićanic, Failure criteria for quasi-brittle materials in latticetype models, Communications in Numerical Methods in Engineering 19 (2003) 703-713.

[25] P. Rossi, F.-J. Ulm, F. Hachi, Compressive behavior of concrete : physical mechanisms and modeling, Journal of Engineering Mechanics 11 (1996) 1038-1043.

[26] J. G. M. Van Mier, Mode ii fracture localization in concrete loaded in compression, Journal of Engineering Mechanics 135 (2009) 1-8.

[27] M. Terrien, Emission acoustique et comportement mécanique post-critique d'un béton sollicité en traction, BLPC 105 (1980) 65-72.

[28] E. Morice, Fissuration dans les matériaux quasi-fragiles : approche numérique et expérimentale pour la détermination d'un modèle incrémental à variables condensées, Ph.D. thesis, École normale supérieure de Cachan (2014).

[29] E. Roubin, Meso-scale fe and morphological modeling of heterogeneous media : applications to cementitious materials, Ph.D. thesis, École normale supérieure de Cachan (2013).

[30] G. A. D'Addetta, Discrete models for cohesive frictional materials, Ph.D. thesis, Universität Stuttgart (2004).

[31] R. A. Vonk, Softening of concrete loaded in compression, PhD thesis, Eindhoven University of Technology, The Netherlands.

[32] M. Vassaux, F. Ragueneau, B. Richard, A. Millard, Compressive behavior of a lattice discrete element model for quasi-brittle materials, Computational Modelling of Concrete Structures 1 (2014) 335-344. 
[33] Z. He, L. Dormieux, E. Lemarchand, D. Kondo, Cohesive mohr-coulomb interface effects on the strength criterion of materials with granular-based microstructure, European Journal of Mechanics - A/Solids 42 (0) (2013) $430-440$.

[34] C. Oliver-Leblond, A. Delaplace, F. Ragueneau, B. Richard, Non-intrusive global/local analysis for the study of fine cracking, International Journal for Numerical and Analytical Methods in Geomechanics 37 (8) (2013) 973-992.

[35] A. Delaplace, R. Desmorat, Discrete 3d model as complimentary numerical testing for anisotropic damage, International Journal of Fracture 148 (2007) $115-128$.

[36] L. Snozzi, A. Caballero, J.-F. Molinari, Influence of the meso-structure in dynamic fracture simulation of concrete under tensile loading, Cement and Concrete Research 41 (11) (2011) 1130-1142.

[37] G. Cusatis, A. Mencarelli, D. Pelessone, J. Baylot, Lattice discrete particle model (ldpm) for failure behavior of concrete. ii: Calibration and validation, Cement Concrete Comp. 33 (9) (2011) 891-905.

[38] D. Grégoire, L. B. Rojas-Solano, G. Pijaudier-Cabot, Failure and size effect for notched and unnotched concrete beams, International Journal for Numerical and Analytical Methods in Geomechanics 37 (10) (2013) 14341452.

[39] C. Le Bellégo, J.-F. Dubé, G. Pijaudier-Cabot, B. Gérard, Calibration of nonlocal damage model from size effect tests, European Journal of Mechanics A/Solids 22 (2003) 3346.

[40] C. G. Hoover, Z. P. Bazant, Cohesive crack, size effect, crack band, International Journal of Fracture 187 (1) (2014) 133-143.

[41] A. Hillerborg, M. Modeer, P. E. Petersson, Analysis of crack formation and crack growth in concrete by means of fracture mechanics and finite elements, Cement and Concrete Research 6 (1976) 773-782. 
[42] M. D. Kotsovos, Effect of testing techniques on the post-ultimate behaviour of concrete in compression, Materiaux et construction 16 (1) (1983) 3-12.

[43] E. Schlangen, Experimental and numerical analysis of fracture processes in concrete, Ph.D. thesis, T.U. Delft, The Netherlands (1993).

[44] M. G. D. Geers, R. De Borst, R. H. J. Peerlings, Damage and crack modeling in single-edge and double-edge notched concrete beams, Engineering Fracture Mechanics 65 (2-3) (2000) 247-261.

[45] R. C. Fenwick, T. Paulay, Mechanisms of shear resistance of concrete beams, Journal of the Structural Division 94 (1968) 2235-2350.

[46] J. C. Walraven, Aggregate interlock : a theoretical and experimental analysis, Thèse de doctorat de l'Université Technologique de Delft, The Netherlands.

[47] D. O. Potyondy, P. A. Cundall, A bonded-particle model for rock, International Journal of Rock Mechanics and Mining Sciences 41 (8) (2004) $1329-1364$.

[48] X. Jourdain, J.-B. Colliat, C. De Sa, F. Benboudjema, F. Gatuingt, Upscaling permeability for fractured concrete: meso-macro numerical approach coupled to strong discontinuities, International Journal for Numerical and Analytical Methods in Geomechanics 38 (5) (2014) 536-550.

[49] A. Tognevi, Modélisation multi-échelle et simulation du comportement thermo-hydro-mécanique du béton avec représentation explicite de la fissuration, Ph.D. thesis, École normale supérieure de Cachan (2012). 